\title{
Double-Grating Displacement Structure for Improving the Light Extraction Efficiency of LEDs
}

\author{
Zhibin Wang, ${ }^{1}$ Yang Hao, ${ }^{1}$ Zhongdong Wang, ${ }^{2}$ Xian Liu, ${ }^{1}$ Qian Zhang, ${ }^{1}$ and Dandan Zhu' \\ ${ }^{1}$ College of Electrical Engineering, Yanshan University, Qinhuangdao 066004, China \\ ${ }^{2}$ Mechanical Science and Engineering Institute, Northeast Petroleum University, Daqing 163318, China
}

Correspondence should be addressed to Zhibin Wang, wzb_ysu@ysu.edu.cn

Received 1 September 2012; Accepted 18 September 2012

Academic Editors: B. Chen, T. Han-Song, and C. Zhan-Ming

Copyright (C) 2012 Zhibin Wang et al. This is an open access article distributed under the Creative Commons Attribution License, which permits unrestricted use, distribution, and reproduction in any medium, provided the original work is properly cited.

\begin{abstract}
To improve the light extraction efficiency of light-emitting diodes (LEDs), grating patterns were etched on GaN and silver film surfaces. The grating-patterned surface etching enabled the establishment of an LED model with a double-grating displacement structure that is based on the surface plasmon resonance principle. A numerical simulation was conducted using the finite difference time domain method. The influence of different grating periods for GaN surface and silver film thickness on light extraction efficiency was analyzed. The light extraction efficiency of LEDs was highest when the grating period satisfied grating coupling conditions. The wavelength of the highest value was also close to the light wavelength of the medium. The plasmon resonance frequencies on both sides of the silver film were affected by silver film thickness. With increasing film thickness, plasmon resonance frequency tended toward the same value and light extraction efficiency reached its maximum. When the grating period for the GaN surface was $365 \mathrm{~nm}$ and the silver film thickness was $390 \mathrm{~nm}$, light extraction efficiency reached a maximum of 55\%.
\end{abstract}

\section{Introduction}

Low-carbon economy and green economy have recently become major themes of global development. As a type of green lighting energy, light-emitting diodes (LEDs) have attracted considerable research attention [1]. LEDs present the advantages of energy conservation, environment friendliness, fast response, and long lifetimes. Thus, LEDs lighting systems have been considered to reduce greenhouse gas emissions by replacing fuel-based lighting in the developing world [2]. Not only that, these light sources are extensively used as backlight sources of liquid crystal displays, advertisements, night lights, traffic signals, outdoor lighting, full color displays [3], and biometric devices [4], in addition, the array of LEDs has been used to inactivate bacteria in liquid suspension and on exposed surfaces [5], among other applications. Despite these advantages, the development of LED core technology is constrained by the photons produced in the lighting devices. Such a constraint prevents photons from being effectively radiated and converted into available optical power. This problem stems from the inherent structure of solid-state lighting devices: the refractive index of solid semiconductor luminophore is higher than that of the surrounding medium [6]. Thus, the light emitted by the lighting devices easily causes total internal reflection in the interface of semiconductor and air, after which the light retraces the optical path of the luminophore and is converted into heat [7]. This phenomenon makes the LEDs working in high-temperature environments for a long time; it not only wastes energy, but also shortens LED lifetimes. So the improvement of LEDs light extraction efficiency can not only extend lifetime, but also make important contribution to the development of the new green energy.

Foreign and domestic experts have begun improving the structure of LED chips, thereby significantly enhancing light extraction efficiency. These improvements primarily include changing the geometric shape of the chips, reducing the duration of total internal reflection in the chips, and decreasing light energy losses. The methods generally applied in realizing such enhancements are using the inverted pyramid structure $[8,9]$, performing surface roughening $[10]$, constructing a mirror with Bragg grating reflection [11], and creating a two-dimensional photonic crystal structure on the surface of $\mathrm{GaN}$ [12-15]. Using the characteristic of surface 
plasmons (SPs) to enhance the light extraction efficiency of LEDs has recently become a research focus [16]. Compare with a single grating structure of the LED model described in [17]. In the current work, a double-grating displacement structure was constructed for an LED model to improve the light extraction efficiency of LEDs. The structure was realized by etching grating patterns on $\mathrm{GaN}$ and metallic silver film surfaces.

Under certain conditions, the free electrons of metal surfaces produce collective coherent oscillation, which is stimulated by light; the electromagnetic wave at the metal/air interface is called SP $[18,19]$. The internal quantum efficiency and external quantum efficiency of LEDs can be improved by using SPs. The improvement in the internal quantum efficiency of LEDs by applying SPs is based on a principle that is related to the spontaneous radiative rate of excitons and state density [20]. When the light center is located in the micro cavity of the wavelength scale, the state density of photons changes, which, in turn, causes variations in the spontaneous radiation rate of excitons. The principle governing the use of SPs to improve the external quantum efficiency of LEDs is based on the observation that light can excite SPs. The light reflection angle is greater than the total internal reflection angle, but the former prevents light from radiating outward. A reasonable metal structure can excite SPs, which are then radiated outward in the form of light. Part of the SPs can be coupled to the electromagnetic waves on the surface of metal, and radiated outward in the form of light by etching a grating-coupled structure on the surface. Part of the incident light that causes total internal reflection can be diminished by grating-patterned etching of the GaN surface. Reducing total internal reflection can also be realized by using a dielectric material with a high coefficient. Using this material produces an evanescent field in total internal reflection. The evanescent field is used as the excitation source that stimulates the SP resonance. It can effectively extract a limited amount of light and reduce the junction temperature of LED chips. Compared with the traditional LED structure, the grating etched onto the GaN surface is relatively complex when used without system optimization. In this work, therefore, the grating period for $\mathrm{GaN}$ and metallic silver film thickness was optimized. The influence of these parameters on light extraction efficiency was also simulated and analyzed.

\section{Theories and Methods}

2.1. Introduction to SP Resonance. When the wave frequency for the excitation of SPs is larger than the plasmon resonance frequency $\left(\omega_{p}\right)$, the electromagnetic field is radiatively propagated far from the space between the metal/dielectric material interface, a phenomenon described as the radiative mode of SPs $[20,21]$. Only when the horizontal direction wave vector $\mathbf{k}_{x}$ is very small (i.e., resonance frequency $\approx \omega_{p}$ ) can the life cycle of the radiant mode of SPs on a metal surface be defined. The gradual increase in $\mathbf{k}_{x}$ results in a resonance mode life cycle that is too short to have any practical significance. The SP resonance in the range of visible frequency

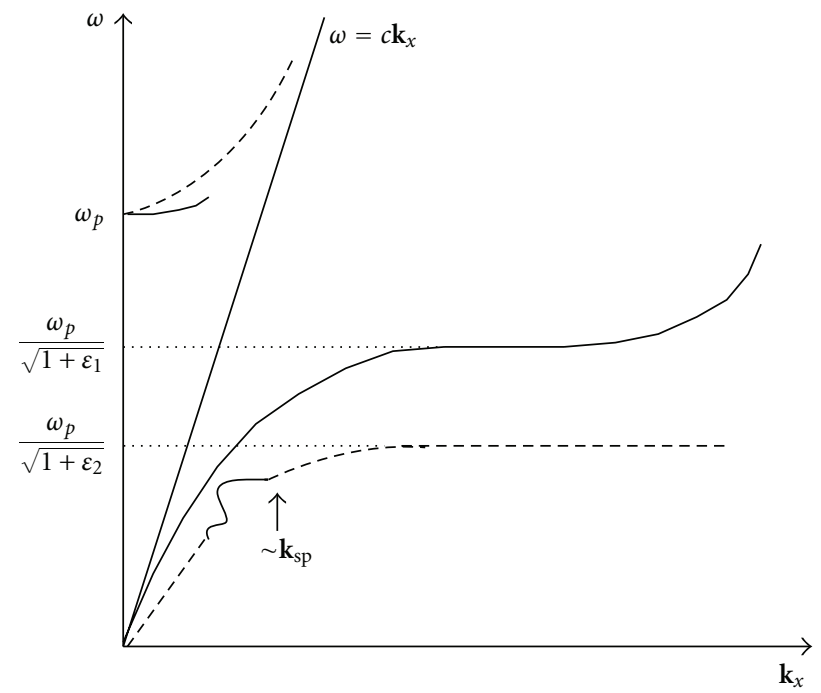

$\omega$ : Incident light frequency.

$c$ : Light velocity.

$\varepsilon_{1} \varepsilon_{2}$ : Material dielectric constant.

FIGURE 1: Dispersion relationship curve of SPs.

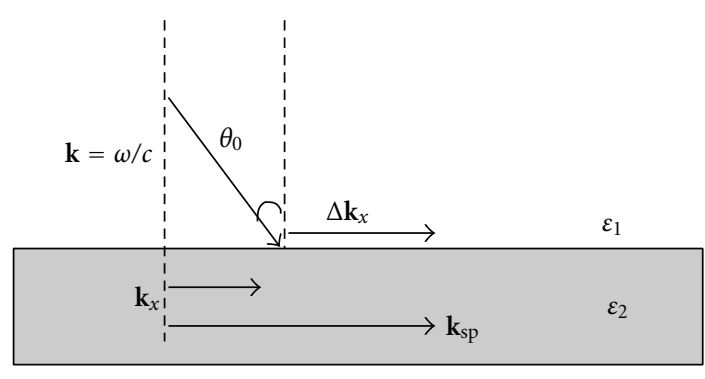

k: Incident light vector

$\theta_{0}$ : Considered angle of incident light and normal line

FIGURE 2: SPs excited by light.

is the nonradiative SP mode. The electromagnetic field produced by this mode is limited to an area near the metal surface. This phenomenon is called electromagnetic wave dispersion.

The SP dispersion relationship is depicted in Figure 1. The dispersion curve of the nonradiative SPs produced on a metal surface lies completely on the right side of the incident electromagnetic wave line of the dielectric material [20]. This result indicates that the SPs have a longer horizontal component of wave vector $\mathbf{k}_{\mathrm{sp}}$ than that of the incident electromagnetic wave vector $\mathbf{k}_{x}$ of the same energy. Thus, the general electromagnetic waves incident from the dielectric material cannot stimulate nonradiative SPs and reach the resonance mode. To match the excitation conditions for the SP resonance mode, a coupled mechanism should be applied. This mechanism increases wave vector $\Delta \mathbf{k}_{x}$ and causes the incident electromagnetic wave to achieve a higher $\mathbf{k}_{x}$ (Figure 2).

Two coupled mechanisms are commonly used [20]. One involves producing a small-raster periodic structure on 
a metal surface as a coupled medium. Under an external electromagnetic field, the free electrons of the metal surface in these cycle structures cause polarized electron oscillation for a specific wavelength. The generated electromagnetic field can provide an additional value $\Delta \mathbf{k}_{x}$ to the incident electromagnetic wave. Subsequently, SP resonance is stimulated. The other coupled mechanism uses the total internal reflection dissipation field produced in a material with a high dielectric constant as the excitation source to stimulate SP resonance.

The electromagnetic wave vector can be written as

$$
\mathbf{k}^{2}=\frac{\omega^{2} \varepsilon \mu}{c^{2}}
$$

where $\varepsilon$ and $\mu$ are the relative permittivity and relative permeability of the material, respectively. When the electromagnetic wave passes through the prism of high permittivity, the wave vector is greater than that observed in dielectric material $\varepsilon_{1}$. When the incident light produces total internal reflection in the prism/dielectric material interface, part of the evanescent field is tunneled into the dielectric material near the total internal reflection interface. The wave vector $\mathbf{k}_{x}$ of the evanescent field and the total internal reflection wave vector have the same size when the distance between the prism and the metal surface is sufficiently small. At the same time, when the incident light wave vector horizontal component of total internal reflection satisfies the conditions of SPs, the SPs in the dielectric material/metal interface are stimulated.

2.2. Finite Difference Time Domain Method. The finite difference time domain (FDTD) method [22], an extension of the finite difference method, is a numerical analysis approach that directly performs computer simulations by using Maxwell equations for electromagnetic fields. Using the Maxwell curl equations in accordance with the field quantities that have special configurations in the Yee grid yields $[23,24]$

$$
\begin{aligned}
\frac{\varepsilon(i+1 / 2, j, k)\left[\mathbf{E}_{x}^{n+1}(i+1 / 2, j, k)-\mathbf{E}_{x}^{n}(i+1 / 2, j, k)\right]}{\Delta t} & \\
+ & \frac{\sigma(i+1 / 2, j, k)\left[\mathbf{E}_{x}^{n+1}(i+1 / 2, j, k)+\mathbf{E}_{x}^{n}(i+1 / 2, j, k)\right]}{2} \\
= & \frac{\left[\mathbf{H}_{z}^{n+1 / 2}(i+1 / 2, j+1 / 2, k)-\mathbf{H}_{z}^{n+1 / 2}(i+1 / 2, j-1 / 2, k)\right]}{\Delta y} \\
& -\frac{\left[\mathbf{H}_{y}^{n+1 / 2}(i+1 / 2, j, k+1 / 2)-\mathbf{H}_{y}^{n+1 / 2}(i+1 / 2, j, k-1 / 2)\right]}{\Delta z} .
\end{aligned}
$$

Let

$$
\begin{array}{r}
p=\left(i+\frac{1}{2}, j, k\right), \quad q^{+}=\left(i+\frac{1}{2}, j+\frac{1}{2}, k\right), \\
q^{-}=\left(i+\frac{1}{2}, j-\frac{1}{2}, k\right), \\
r^{+}=\left(i+\frac{1}{2}, j, k+\frac{1}{2}\right), \quad r^{-}=\left(i+\frac{1}{2}, j, k-\frac{1}{2}\right) .
\end{array}
$$

Thus,

$$
\begin{aligned}
\left(\mathbf{E}_{x}\right)_{p}^{n+1}= & \frac{\left[1-\Delta t \sigma_{p} / 2 \varepsilon_{p}\right]\left(\mathbf{E}_{x}\right)_{p}^{n}}{\left[1+\Delta t \sigma_{p} / 2 \varepsilon_{p}\right]} \\
& +\frac{\left(\Delta t / \varepsilon_{p}\right)\left\{\left[\begin{array}{l}
{\left[\left(\mathbf{H}_{z}\right)_{q^{+}}^{n+1 / 2}-\left(\mathbf{H}_{z}\right)_{q^{-}}^{n+1 / 2}\right] / \Delta y-} \\
{\left[\left(\mathbf{H}_{y}\right)_{r^{+}}^{n+1 / 2}-\left(\mathbf{H}_{y}\right)_{r^{-}}^{n+1 / 2}\right] / \Delta z}
\end{array}\right\}\right.}{\left[1+\Delta t \sigma_{p} / 2 \varepsilon_{p}\right]},
\end{aligned}
$$

where the superscripts $n+1 / 2$ and $n$ are the time stepnumbers; a group of changes $(i, j, k)$ and $\Delta t$ are the time steps; $\Delta x, \Delta y, \Delta z$ are the distances of the adjacent lattice point in the $x, y, z$ directions, respectively. Other field quantities can be treated in the same manner.

To perform a stable numerical simulation, the variables should satisfy

$$
v_{\max } \Delta t \leq \frac{1}{\sqrt{(1 / \Delta x)^{2}+(1 / \Delta y)^{2}+(1 / \Delta z)^{2}}},
$$

where $v_{\max }$ is the maximum phase velocity, $\Delta t$ denotes the time step, and $\Delta x, \Delta y, \Delta z$ are the distance steps in the $x, y, z$ directions, respectively. When $\Delta x=\Delta y=\Delta z=\Delta$, (5) can be simplified as $v_{\max } \Delta t<\Delta / \sqrt{3}$. To reduce the error caused by numerical dispersion effects, (5) should also satisfy the conditions $\Delta / \lambda_{\min }<1 / 10$, where $\lambda_{\min }$ is considered the shortest wavelength of the electromagnetic wave considered.

The FDTD method was used to solve the numerical analysis problems presented by grating-patterned etching on the metallic silver film. This approach also enables the excitation of SPs and application of the total internal reflection dissipation field for exciting the SPs. The changes in time step $\Delta t$ reflect the variations in energy flow on the receiving surface. This reflection is realized by placing a receiving surface over the structure. The changes in energy flow facilitate the analysis of the effects of structural parameter variations on the light extraction efficiency of LEDs.

\section{Physical LED Model}

The physical model of the LED with a double-grating displacement structure was constructed by grating-patterned etching on the $\mathrm{GaN}$ and metallic silver film surfaces (Figure 3). The model comprises the silver film layer, P-GaN layer, active layer, $\mathrm{N}-\mathrm{GaN}$ layer, and $\mathrm{SiC}$ substrate. During the experiment, the calculated area dimensions of $4200 \mathrm{~nm} \times$ $420 \mathrm{~nm}$ were realized; the thicknesses of the P-GaN and NGaN layers were 200 and $400 \mathrm{~nm}$, respectively. Compare with the physical LED model as described in [17], a gratingpatterned etching was added to the GaN surface, which had two reasons. (1) Grating-patterned etching can diminish part of the total internal reflection produced by incident light. (2) A portion of the light causes the evanescent field of total internal reflection to excite SPs; The study in [7] has discussed that grating-patterned etching on the top surface can effectively enhance the light extraction efficiency of LEDs; thus, this phenomenon results in a combined effect with 


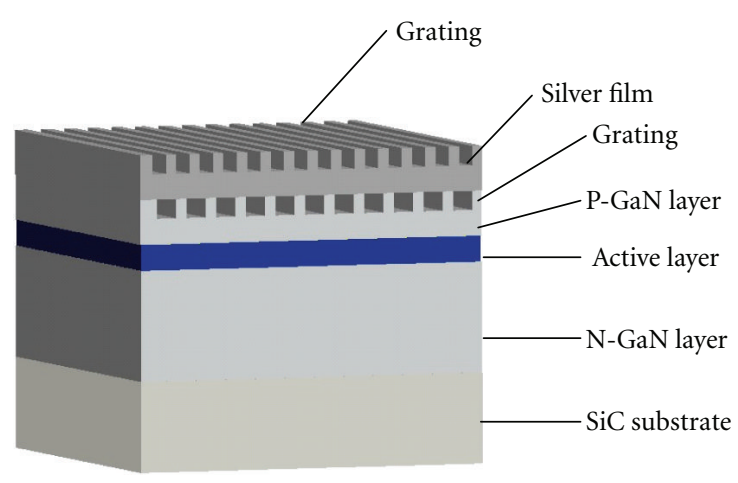

FIgure 3: Physical model of an LED with a double-grating displacement structure.

grating-patterned etching on the metallic silver film surface, thereby enhancing optical transmission and improving the light extraction efficiency of LEDs.

\section{Numerical Simulation and Analysis}

A numerical simulation was conducted using the FDTD method. The wavelength of light was $500 \mathrm{~nm}$, the refractive index of air was 1.0, and the refractive index of $\mathrm{GaN}$ was 2.4. The silver film was used in the Lorentz dispersion model. In analyzing the light absorption process, the silver film is disregarded. The light emitted by the active layer is replaced by the total field-scattered field source, an approach that realizes incident plane waves from all directions. The intensity was 1 and the grating period of the metallic silver film was $375 \mathrm{~nm}$. A receiving surface, which is capable of reflecting light intensity, was placed above the model to keep track of energy flow.

\subsection{Effect of Grating Period on the Light Extraction Efficiency} of the GaN Surface. Figure 4 presents the energy flow of the $\mathrm{GaN}$ with a double-grating displacement structure at different grating periods. The maximum flow intensity value was 0.44 , which can be obtained at a GaN surface grating period of $365 \mathrm{~nm}$. Figure 5 shows the relationship between the surface grating period of $\mathrm{GaN}$ and light extraction efficiency when the silver film thickness changes from $300 \mathrm{~nm}$ to $400 \mathrm{~nm}$. For light extraction efficiency with oscillatory changes in grating period, the maximum value acquired constantly ranged from $360 \mathrm{~nm}$ to $380 \mathrm{~nm}$. This result confirms that grating period influences the light extraction efficiency of LEDs. At a silver film thickness of $300 \mathrm{~nm}$ to $400 \mathrm{~nm}$, the relationship curves of grating period and light extraction efficiency are similar and the maximum light extraction efficiency occurred at a $365 \mathrm{~nm}$ grating period. When the grating period continued to increase, the light extraction efficiency gradually declined. This result is attributed to two factors. First, the surface grating period is related to the wave vector that causes plasmon resonance. Thus, only when the grating period satisfies the excitation conditions can the SPs be excited. Consequently, visible light

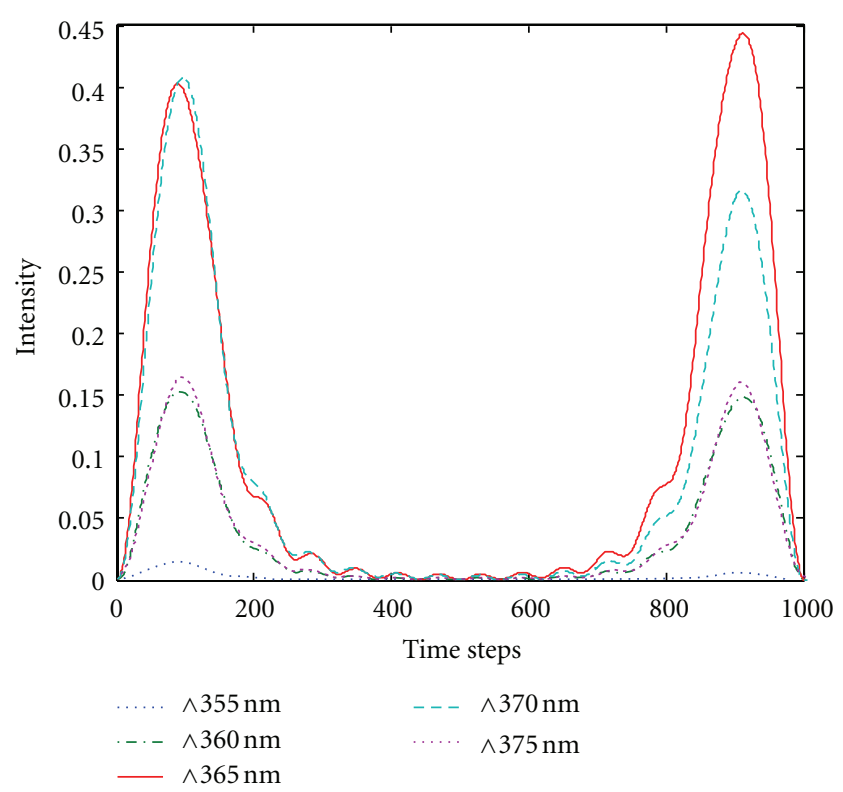

FIgURE 4: Energy flow of GaN at different grating periods.

is extracted and transmissible light is enhanced. Second, the ideal size of diffraction grating satisfies the wavelength of light on a metal surface when the period is considerably smaller than the wavelength of light. Otherwise, conductivity to light diffraction is low and the effect of changing light directions caused by light diffraction decreases. On the other hand, when the period is far from the wavelength of light, the plane of the grating section is excessively large, thereby causing stronger total internal reflection, which slows down light emission.

4.2. Effect of Silver Film Thickness on Light Extraction Efficiency. The grating period was set at $365 \mathrm{~nm}$ on the basis of the analysis in Section 4.1. The thickness of the metallic silver film was set at $300 \mathrm{~nm}$ to $400 \mathrm{~nm}$. Figures 6 and 7 show the energy flow of the silver film with a double-grating displacement structure and that of the silver film with a single grating, respectively. The light extraction efficiency of LEDs with a double-grating displacement structure improved by $43 \%$ over that of the silver film surface with a single grating. The graph shows that the silver film thickness should satisfy the grating coupling conditions to enhance light extraction efficiency. A silver film that is too thick or too thin cannot induce coupling. The plasmon resonance mode existed on both sides of the metal/dielectric material interface. Thus, when the film thickness was at the nanometer scale, the evanescent field formed by the SPs was strong enough to pass through the other side of the metal. Consequently, the SP electromagnetic fields on both sides of the metal film interacted with each other and formed a group of coupled SPs, as illustrated by the energy flow curve of silver thickness at $390 \mathrm{~nm}$ in Figure 6.

The plasmon resonance frequency reached the same value and energy flow intensity reached a maximum of 0.55 . 


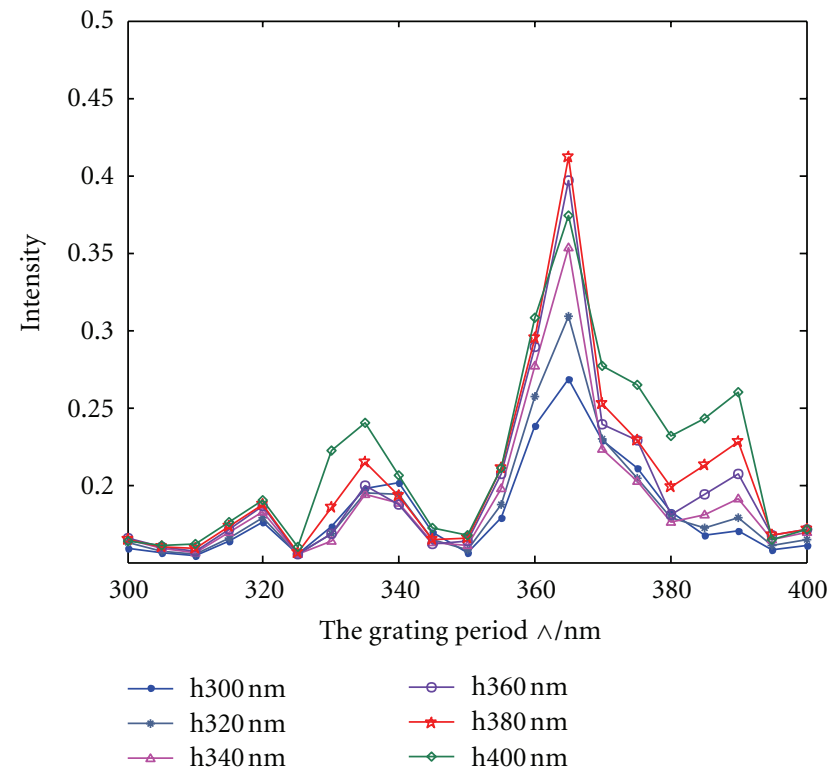

FIGURE 5: Relationship between the grating period of GaN and light extraction efficiency with silver film thickness ranging from $300 \mathrm{~nm}$ to $400 \mathrm{~nm}$.

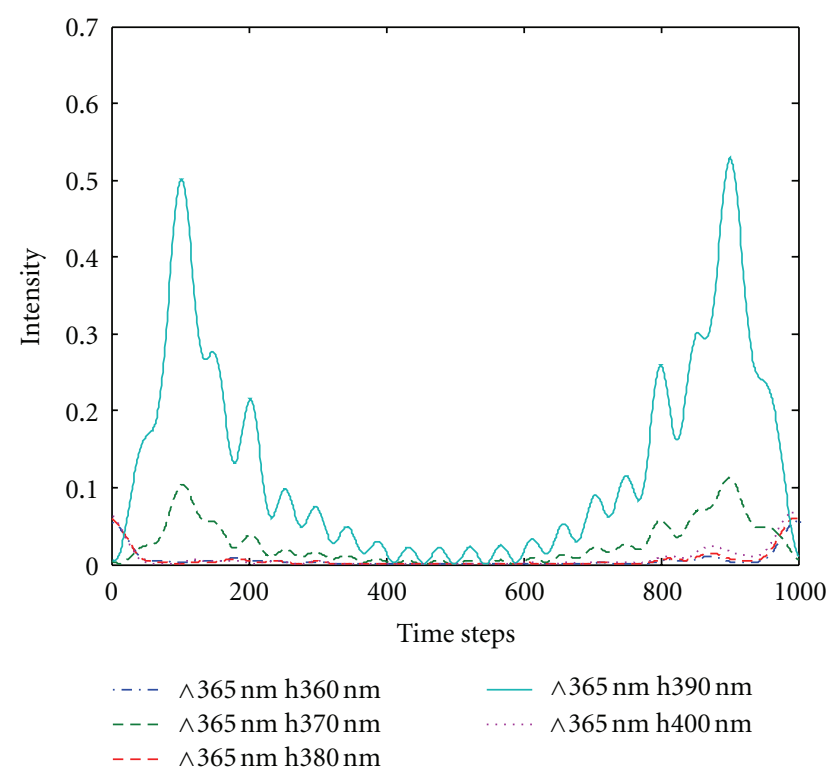

Figure 6: Energy flow of silver film (of different thicknesses) with a double-grating structure.

However, when the silver film thickness was smaller than $390 \mathrm{~nm}$, the SP resonance frequency remained at the degenerate state, in which the energy flow intensity exhibited no significant enhancement. The results indicate the absence of a coupled phenomenon. With increasing silver film thickness, the energy flux rate gradually decreased, indicating the continuous division of the SP resonance frequency. Consequently, coupling disappeared when the silver film thickness tended toward infinity.

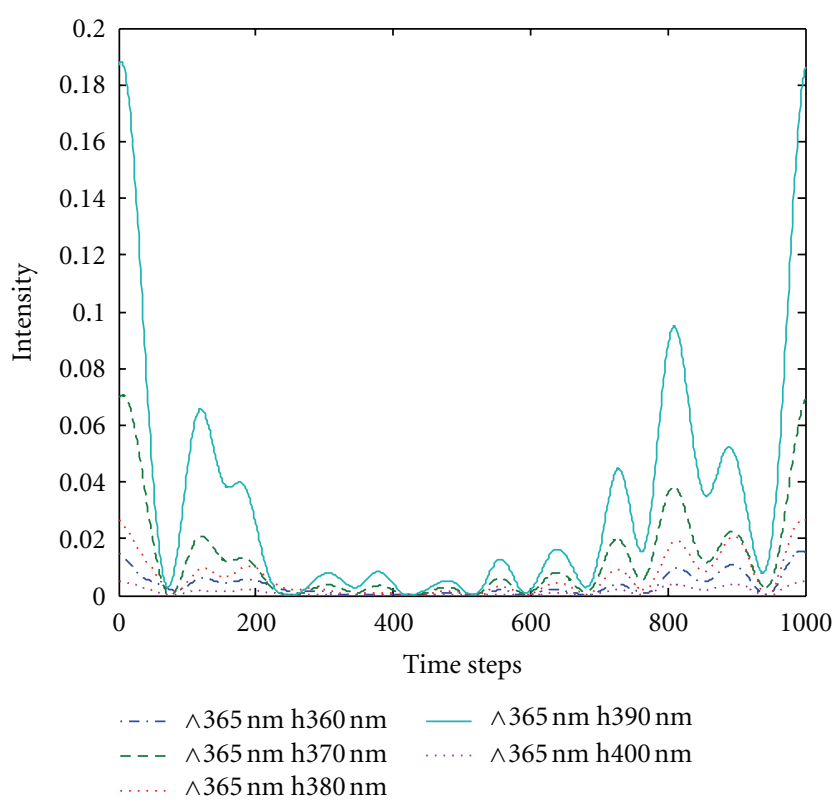

FIGURE 7: Energy flow of silver film (of different thicknesses) with a single grating structure.

\section{Conclusion}

Grating-patterned etching was performed on $\mathrm{GaN}$ and silver film surfaces to establish an LED model with a doublegrating displacement structure. The FDTD method was used in conducting a numerical simulation. A grating etched on the GaN surface effectively extracts a limited amount of light and enhances the light extraction efficiency of LEDs. At a GaN grating period of $365 \mathrm{~nm}$ and a silver film thickness of $390 \mathrm{~nm}$, light extraction efficiency reaches a maximum of $55 \%$.

\section{Acknowledgments}

This work was supported by the National Natural Science Foundation of China under Grant no. 61107039 and no. 61004050 and the Natural Science Foundation of Heilongjiang Province under Grant no. F201006.

\section{References}

[1] X. Gu, T. Qiu, W. Zhang, and P. K. Chu, "Light-emitting diodes enhanced by localized surface plasmon resonance," Nanoscale Research Letters, vol. 6, no. 1, article 199, 2011.

[2] E. Mills and A. Jacobson, "From carbon to light: a new framework for estimating greenhouse gas emissions reductions from replacing fuel-based lighting with LED systems," Energy Efficiency, vol. 4, no. 4, pp. 523-546, 2011.

[3] H. Lin, S. Liu, X. S. Zhang, B. L. Liu, and X. C. Ren, "Enhanced external quantum efficiency of light emitting diodes by fabricating two-dimensional photonic crystal sapphire substrate with holographic technique," Acta Physica Sinica, vol. 58, no. 2, pp. 959-963, 2009. 
[4] N. Kourkoumelis and M. Tzaphlidou, "Eye safety related to near infrared radiation exposure to biometric devices," TheScientificWorldJournal, vol. 11, pp. 520-528, 2011.

[5] L. E. Murdoch, M. MacLean, E. Endarko, S. J. MacGregor, and J. G. Anderson, "Bactericidal effects of 405nm light exposure demonstrated by inactivation of escherichia, salmonella, Shigella, Listeria, and mycobacterium species in liquid suspensions and on exposed surfaces," The Scientific World Journal, vol. 2012, Article ID 137805, 8 pages, 2012.

[6] D. H. Kim, C. O. Cho, Y. G. Roh et al., "Enhanced light extraction from GaN-based light-emitting diodes with holographically generated two-dimensional photonic crystal patterns," Applied Physics Letters, vol. 87, no. 20, Article ID 203508, 3 pages, 2005.

[7] S. Trieu, X. Jin, A. Ellaboudy et al., "Top transmission grating GaN LED simulations for light extraction improvement," in Physics and Simulation of Optoelectronic Devices XIX, vol. 7933 of Proceedings of SPIE, San Francisco, Calif, USA, January 2011.

[8] C. M. Tsai, J. K. Sheu, W. C. Lai et al., "Enhanced output power in GaN-based LEDs with naturally textured surface grown by MOCVD," IEEE Electron Device Letters, vol. 26, no. 7, pp. 464466, 2005.

[9] W. Zhou, G. Min, Z. Song, J. Zhang, Y. Liu, and J. Zhang, "Enhanced efficiency of light emitting diodes with a nano-patterned gallium nitride surface realized by soft UV nanoimprint lithography," Nanotechnology, vol. 21, no. 20, Article ID 205304, 2010.

[10] K. S. Kim, S. M. Kim, H. Jeong, M. S. Jeong, and G. Y. Jung, "Enhancement of light extraction through the wave-guiding effect of $\mathrm{ZnO}$ sub-microrods in InGaN blue light-emitting diodes," Advanced Functional Materials, vol. 20, no. 7, pp. 1076-1082, 2010.

[11] C. M. Lim and G. Hugh Song, "Design of superperiodic photonic-crystal light-emitting plates with highly directive luminance characteristics," Journal of the Optical Society of America B, vol. 26, no. 2, pp. 328-336, 2009.

[12] A. David, H. Benisty, and C. Weisbuch, "Optimization of lightdiffracting photonic-crystals for high extraction efficiency LEDs," IEEE/OSA Journal of Display Technology, vol. 3, no. 2, pp. 133-148, 2007.

[13] D. H. Long, I. K. Hwang, and S. W. Ryu, "Design optimization of photonic crystal structure for improved light extractionof GaN LED," IEEE Journal on Selected Topics in Quantum Electronics, vol. 15, no. 4, pp. 1257-1263, 2009.

[14] A. I. Zhmakin, "Enhancement of light extraction from light emitting diodes," Physics Reports, vol. 498, no. 4-5, pp. 189 $241,2011$.

[15] T. A. Truong, L. M. Campos, E. Matioli et al., "Light extraction from GaN-based light emitting diode structures with a noninvasive two-dimensional photonic crystal," Applied Physics Letters, vol. 94, no. 2, Article ID 023101, 33 pages, 2009.

[16] V. K. Komarala, W. H. Guo, and M. Xiao, "Surface plasmon density of states at the metal-dielectric interface: dependence of metal layer thickness and dielectric material," Journal of Applied Physics, vol. 107, no. 1, Article ID 014309, 5 pages, 2010.

[17] Y. Z. Lin, K. Li, F. M. Kong, J. Zhao, L. G. Du, and H. Gao, "Comprehensive numeric study of gallium nitride light-emitting diodes adopting surface-plasmon-mediated light emission technique," IEEE Journal on Selected Topics in Quantum Electronics, vol. 17, no. 4, pp. 942-951, 2010.

[18] Z. Wu, J. W. Haus, Q. Zhan, and R. L. Nelson, "Plasmonic notch filter design based on long-range surface plasmon excitation along metal grating," Plasmonics, vol. 3, no. 2-3, pp. 103-108, 2008.

[19] S. H. Choi, S. J. Kim, and K. M. Byun, "Characteristics of light emission from surface plasmons based on rectangular silver gratings," Optics Communications, vol. 283, no. 14, pp. 29612966, 2010.

[20] H. Raether, Surface Plasmons on Smooth and Rough Surface and on Gratings, Springer, Berlin, Germany, 1998.

[21] S. Glasberg, A. Sharon, D. Rosenblatt, and A. A. Friesem, "Long-range surface plasmon resonances in grating-waveguide structures," Applied Physics Letters, vol. 70, no. 10, pp. 1210-1212, 1997.

[22] K. S. Yee and J. S. Chen, "The finite-difference time-domain (FDTD) and the finite-volume time-domain (FVTD) methods in solving Maxwell's equations," IEEE Transactions on Antennas and Propagation, vol. 45, no. 3, pp. 354-363, 1997.

[23] X. Zhou and W. Lin, "Numerical stability and numerical dispersion of compact conformal mapping 2D-FDTD method used for the dispersion analysis of waveguides," International Journal of Infrared and Millimeter Waves, vol. 20, no. 7, pp. 1403-1412, 1999.

[24] M. T. Bettencourt, "Flux limiting embedded boundary technique for electromagnetic FDTD," Journal of Computational Physics, vol. 227, no. 6, pp. 3141-3158, 2008. 

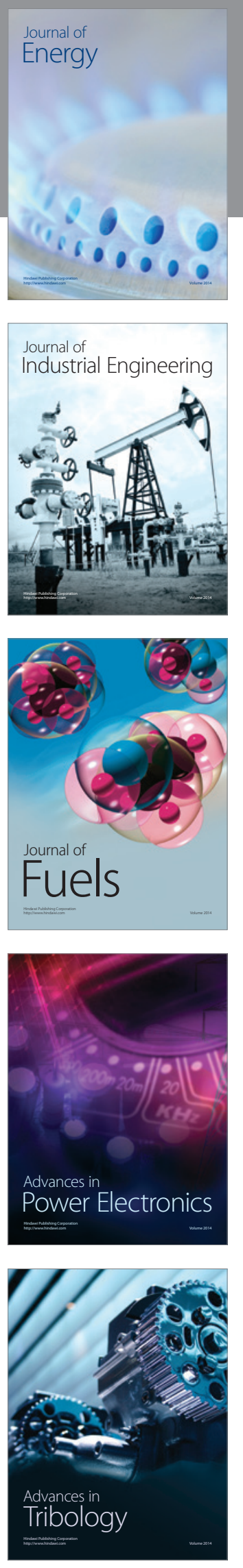
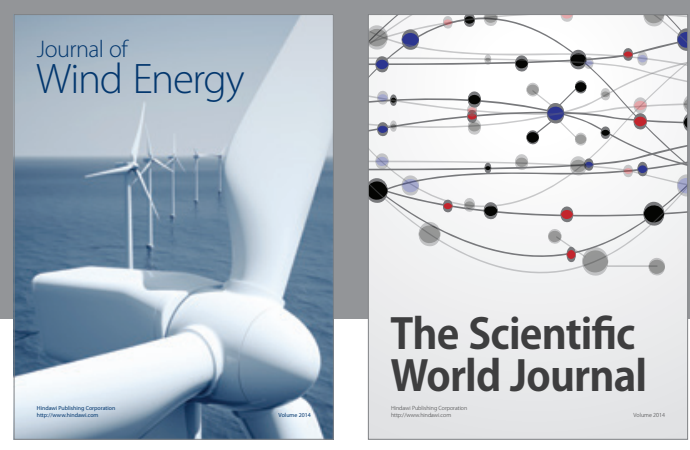

The Scientific World Journal

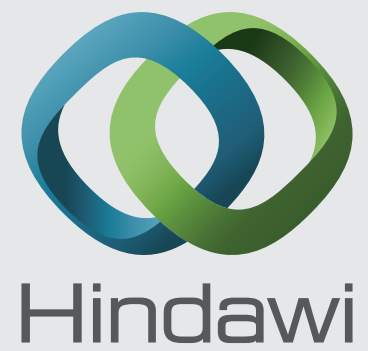

Submit your manuscripts at http://www.hindawi.com
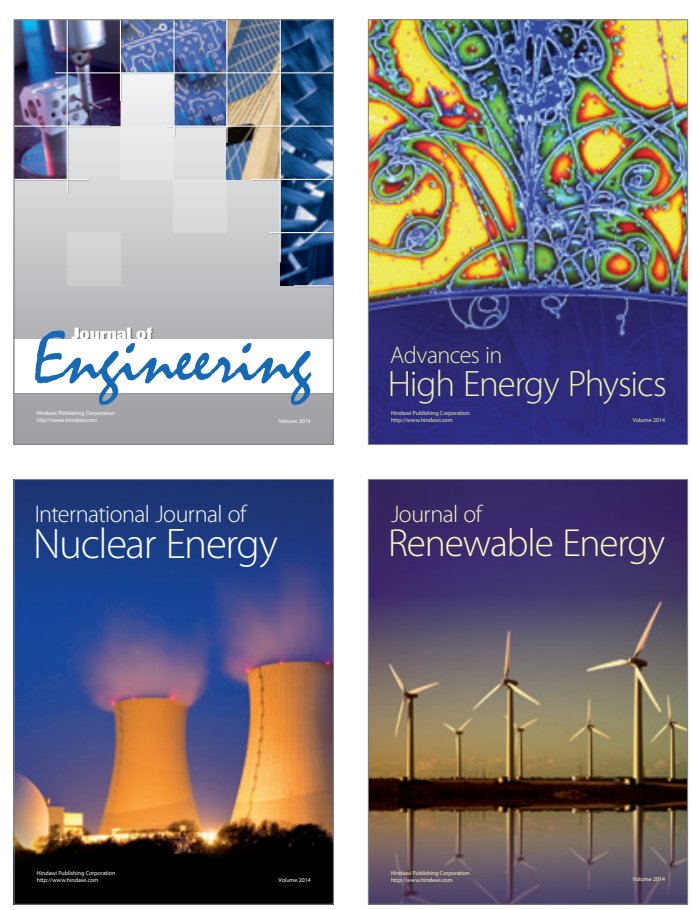

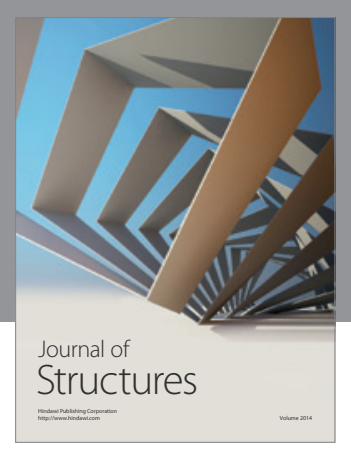

Rotating
Mechinery
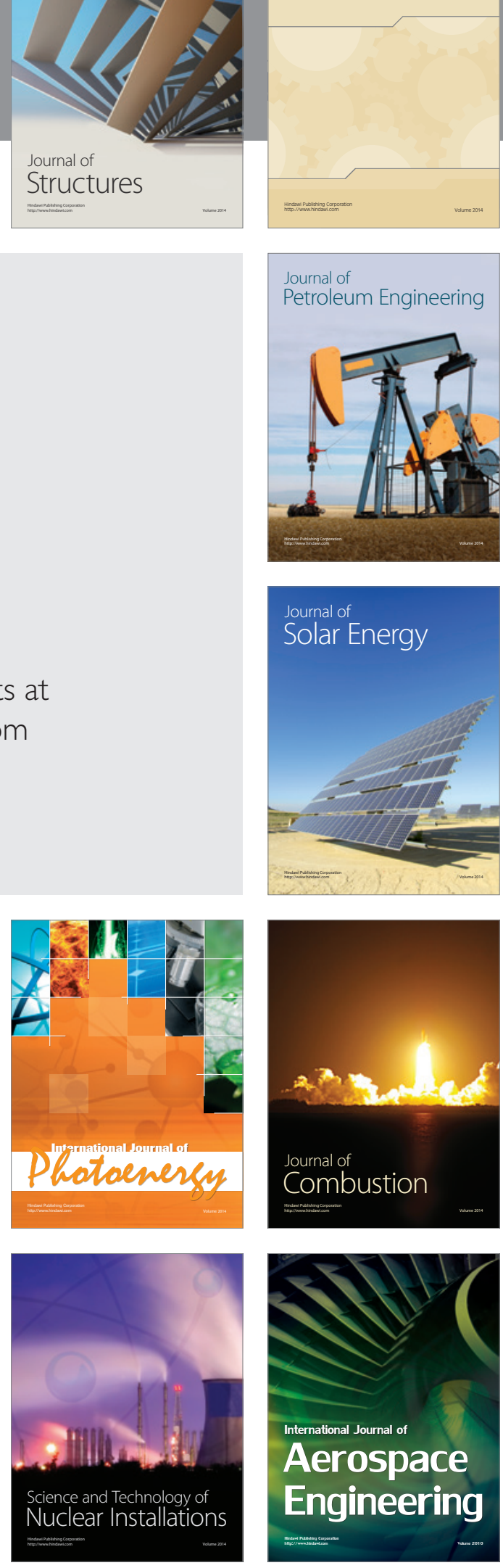